\title{
Gravitational self-regularization of quantum fields at Planck scales
}

\author{
Zahid Zakir
}

\begin{abstract}
Loop diagrams with near-Planck energies create a strong external gravitational field, which slows down local processes for distant observers up to their freezing. Since Planck length is the gravitational radius of the system of quanta, the events of this and smaller scale cannot occur in finite world time $t$ and do not contribute to the S-matrix. Consequently, gravitational time dilation, leading to a strong redshift of local frequencies, provides gravitational self-regularization of the loop diagrams. The loop corrections without gravity effects, cut off at Planck energy, give upper bounds for the corrections with gravity effects and this fact leads to simple rules of gravitational regularization. The corrections with quanta of gauge fields and gravitons are small, and the perturbation theory series converge. At pre-Planck energies, oneloop graviton contributions are sufficient, since the multi-loop ones are damped by high degrees of the relation "energy/Planck energy". Scalar field with power-law growing corrections should be effective field. Non-linearity of fields enhances gravity and get faster freezing, which suppresses the high energy terms. Nonrenormalizable models are finite, but become consistent only when their loop corrections remain small on Planck scale and this occurs in quantum gravity. Gravitationally regularized Extended Standard Model (ESM), including gravitons and Standard Model with effective scalars, is renormalizable and finite, which simplifies its further generalization.
\end{abstract}

Keywords: Gravitational redshift; quantum fields; regularization; renormalization; quantum gravity

\section{Content}

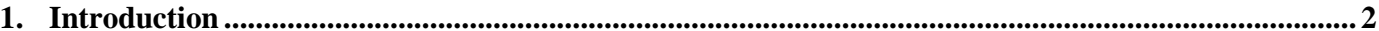

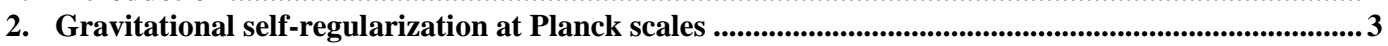

2.1. The problem of ultraviolet divergences and its solution by inclusion of gravity............................. 3

2.2. The invariant gravitational regularization at Planck scales ......................................................... 4

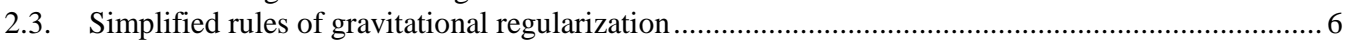

3. Gravitationally-regularized SM and quantum gravity ................................................................................ 7

3.1. Upper limits of the loop corrections in QED ............................................................................. 7

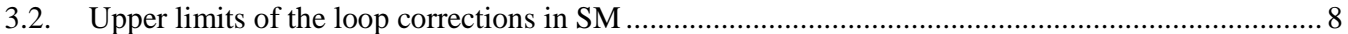

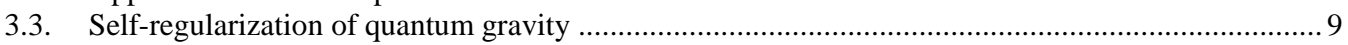

4. Extended SM with gravitons and perspectives of generalization ............................................................... 10

4.1. The finiteness of theories of quantized fields and ESM .......................................................... 10

4.2. Simplification of unification models and perspectives for compositeness ..................................... 10

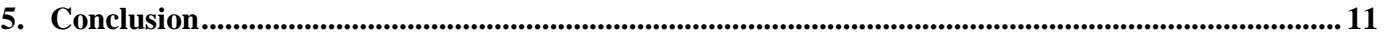

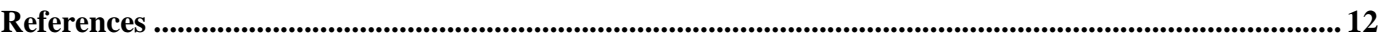

${ }^{1}$ Center for Theoretical Physics and Astrophysics, Tashkent Uzbekistan, zzakir@ qgph.org, ORCID 


\section{Introduction}

Quantum field theory (QFT) in its standard formulation [1,2] contained ultraviolet divergences in loop diagrams, which, in fact, made the theory inconsistent and this fundamental problem remained unsolved. The standard empirical recipe, replacing the diverging theoretical masses and charges with their experimental values, avoids the problem in practice, but does not solve it.

As in classical electrodynamics, divergences arise due to the idealization of point particles. However, general relativity introduces a fundamental lower limit for the size of any system for external observers - its gravitational radius. A system of particles, when its size approaches the Planck length $l_{g}=\left(G h / c^{3}\right)^{1 / 2} \simeq 1.616 \times 10^{-35} \mathrm{~m}$ ( $G$ is the gravitational constant) creates an external gravitational field around its center of inertia. The scale $l_{g}$ is determined by the fact that here the gravitational radius, which is proportional to the energy, equals the quantummechanical wavelength, which is inversely proportional to the energy. Therefore, since $l_{g}$ is the gravitational radius of an object with the Planck energy $\Lambda_{g}=\left(h c^{5} / G\right)^{1 / 2} \simeq 1.221 \times 10^{19} \mathrm{GeV}$, this leads to a strong dilation of local proper times of particles in terms of distant observer's (world) time $t$ and the gravitational redshift of their local frequencies up to vanishing near $l_{g}$ [3]. However, these strong restrictions of general relativity was not satisfied not only in the standard QFT without gravity, but in previous attempts to include gravity in particle physics also [4-11].

As it was shown recently [12], in fact, the problem of finiteness of the loop corrections in QFT has a simple and natural solution at following the above-mentioned general relativistic restriction on physical events imposed by gravitational time dilation [3,12]. In this paper, briefly describing this new approach, simple rules of gravitational regularization will be formulated and some consequences for QFT, including quantum gravity, will be discussed.

Notice, that the situation with gravity in particle physics appears similar to the two-stage history of atomic physics. In the latter, at the first stage, the Coulomb field, together with quantum effects, led to the basic structure of atomic energy levels. Only after that, at the second stage, the effects of photons, quantized (transverse) components of the electromagnetic field, were included, which gave only small corrections to the discrete Coulomb levels (Lamb shift).

However, in particle physics, for historical reasons, these two stages turned out to be rearranged, and so far, inclusion of gravity has mainly been reduced to the second stage accounting for the transverse gravitons. However, the first stage, adequately inclusion of the (longitudinal) external gravitational field (such as the Schwarzschild field), was not passed.

The main result of the present approach is the restoration of the normal sequence of stages, similar to the case of electromagnetic field, for gravity in particle physics also. The first stage, the inclusion of a longitudinal (Schwarzschild or Kerr) gravitational field as an external field, leads to the natural self-regularization of all fields due to gravitational freezing near $l_{g}$. The second stage, the inclusion of (transverse) gravitons, then leads to small corrections. As a result, the Extended SM (ESM), containing the Standard Model (SM) with an effective scalar field and quantum gravity, appear as finite and renormalizable.

In Section 2 of the paper, the rules of gravitational regularization of QFT are formulated. In Section 3 the method is applied to ESM and in Section 4 further perspectives of ESM and its generalizations are discussed. 


\section{Gravitational self-regularization at Planck scales}

\subsection{The problem of ultraviolet divergences and its solution by inclusion of gravity}

In the standard QFT in flat spacetime, the loop diagrams of gauge fields, interacting with fermions, grow with the cutoff energy $\Lambda$ logarithmically $a \ln (\Lambda / m)$, where $m$ is the mass of a light fermion. The coefficients $a$ in the case of SM fields are small $a \ll 1$ and

$$
a \ln \frac{\Lambda_{g}}{m c^{2}}<1, \frac{1}{a}>\ln \frac{\Lambda_{g}}{m c^{2}} .
$$

i.e. the corrections remain small up to Planck energy $\Lambda_{g}$. Further extrapolation to higher cutoff energies $\Lambda>\Lambda_{g}$ then led to the divergences.

At energies of intermediate states close to $\Lambda_{g}$ gravity becomes dominant, but it was included mainly in the form of gravitons, spin 2 quanta. The estimations show that the contribution of gravitons to the $L$-loop diagrams increases with $\Lambda$ as $\sim\left(\Lambda / \Lambda_{g}\right)^{2 L+2}$, i.e. one-loop diagrams grow as $\sim\left(\Lambda / \Lambda_{g}\right)^{4}$ and then each additional loop adds two to the degree of growth. The graviton contributions, therefore, remain small at $\Lambda<\Lambda_{g}$, but diverge catastrophically at $\Lambda \geq \Lambda_{g}$ (see also Section 5.1).

The success of the renormalized QFT in particle physics showed that the cutoff energy $\Lambda$ can not be infinite and there is a certain physical mechanism making it finite. Moreover, only the discovery of such a mechanism would make QFT as a theory consistent from both mathematical and physical points of view. For this reason, there have been many attempts to find this physical mechanism.

The main direction of the search was the inclusion of gravity at $l_{g}$. But attempts in this direction remained in the form of semi-quantitative estimates. Moreover, in any case different reasons for the Planck scale cutoff were indicated, mainly reducing them to the difficulties of observations [4-11]. This was unconvincing, since the lack of contribution to the observables from smaller distances $l \leq l_{g}$ and higher cutoff energies $\Lambda \geq \Lambda_{g}$ had not been proven.

As a result, it became leading the alternative direction of the search based on inventing new radical hypotheses by supposing a rich physics at $l \leq l_{g}$. However, these pure mathematical approaches did not have an experimental basis and led to set of new problems, again requiring new hypotheses.

Thus, on the one hand, numerous attempts to exclude two groups of ultraviolet divergences at $\Lambda \geq \Lambda_{g}$, logarithmic for gauge fields and power-law for gravitons, were unsuccessful. On the other hand, the effects of the external gravitational field of quanta near $\Lambda_{g}$ were practically ignored. As it was shown in [12], these two situations are closely related and the improvement of the second weakness by adequate inclusion of the effects of gravity as an external field generated by the energy of quanta in the loops, solves the first problem by excluding the divergences. Below this approach will be briefly described and then its consequences in particle physics will be considered.

The action function of QFT with the matter field Lagrangian $L_{m}$ must also contains the Lagrangian $L_{g}$ of the gravitational field generated by the energy-momentum tensor $T_{\mu \nu}$ of this matter. This gravitational field is described in general relativity by the non-trivial (i.e. generating by $T_{\mu v}$ ) part of the metric $g_{\mu \nu}$ in the asymptotically flat spacetime with world time 
$t$. The variation of the action function $\delta S / \delta g_{0 \mu}=0$ gives four Einstein equations, which do not contain second time derivatives of $g_{0 \mu}$ and, therefore, represent constraints [11]. As the result, the components $g_{0 \mu}$ remain classical and their non-trivial part describe an external field part of gravity.

In the static frame, where the center of inertia of a source is rested, $g_{00}$, representing an external gravitational field, leads to gravitational time dilation $\Delta \tau=g_{00}^{1 / 2} \Delta t$. For the Schwarzschild field, generated by the source with energy $E$, it is related with the radial (longitudinal) component of the field, as $g_{00}=-g_{11}^{-1}=1-r_{g} / r$, where $r_{g}=2 G E / c^{4}$ is the gravitational radius .

The graviton field $h_{\mu v}$, a small deviation from the transverse part of the background metric $g_{\mu \nu}^{(T)}$, is quantized and, due to non-linearity of the Einstein equations, also generates an external gravitational field. Its contribution to $g_{00}$ further increases the effects of gravitational time dilation, which then lead to the self-regularization of quantum gravity (see Section 5.1)..

\subsection{The invariant gravitational regularization at Planck scales}

In general relativity, there are three types of time dilation - inertial, non-inertial and gravitational. All they are purely relativistic effects and any of them has significant distinctions. The inertial time dilation is relative and symmetric for two standard clocks resting in two inertial frames. The non-inertial time dilation, the dilation of the proper time interval of a noninertial clock with respect to the proper time interval of an inertial clock is absolute and asymmetric for these two clocks, as is well-known from the clock (or twin) paradox. Also irreversible and absolute is the dilation of the proper times of two clocks that have experienced different accelerations - the slower will be the one that accelerated stronger and/or longer. The gravitational time dilation, the dilation of the proper time of a clock resting in a gravitational field with respect to the proper time of a resting distant clock, is similar to the non-inertial case and is also absolute and asymmetric for these two clocks.

For this reason, at describing the gravitational effects near $l_{g}$, especially the dilation of local proper times of quanta in terms of $t$, they should be considered as irreversible and absolute slowdown with respect to the world time $t$, marking the global hypersurfaces of simultaneity where amplitudes are defined.

In the presence of gravity, the scalar field $\phi(t, \mathbf{x})$ and its frequency modes are defined on the hypersurfaces $t=$ const and have the form:

$$
\phi(t, \mathbf{x})=\sum_{\mathbf{p}}\left[a_{\mathbf{p}} f(\mathbf{p}, \mathbf{x}) e^{-i \omega t}+a_{-\mathbf{p}} f^{*}(\mathbf{p}, \mathbf{x}) e^{i \omega t}\right] .
$$

where $\omega=2 \pi v=\left(\mathbf{p}^{2}+m^{2}\right)^{1 / 2}$ is the frequency in terms $t$. In a system of interacting quanta, particularly in a loop diagram, the total energy of the system generates at every moment $t$ an external gravitational field around the center of inertia of the system, which, through $g_{00}$, slows down the local proper times of quanta $\tau$ with respect to $t$. This leads to the gravitational redshift of frequencies $v=v_{0} g_{00}^{1 / 2}$, which is not an apparent effect and the particles are really created with lower frequencies $v<v_{0}$ than the frequencies $v_{0}$ near distant observers. As a result, at small distances, $v$ reaches a maximum value $v_{\max }<v_{g}$ near $l_{g}$ and quickly 
decreases due to the strong gravitational redshift. Here the Planck frequency $v_{g}$ is defined as: $v_{g}=\Lambda_{g} / h$.

In a two-particle system, the gravitational redshift of proper frequencies is small already at distances $\sim 3 l_{g}$ between particles, and at larger distances $r \geq 3 l_{g}$ the effects of gravity are weak and the standard QFT is applicable.

In (2), instead of $v_{0}$, there appear the frequency $v=\omega / 2 \pi$ with strong redshift and, therefore, the contribution to the amplitudes of fluctuations in the interval $l_{g} \leq r \leq 3 l_{g}$ is small and represents a small correction to the main contribution from the region $3 l_{g} \leq r \leq \infty$. Indeed, the localization region of an ultrarelativistic particle is related to its wavelength as $r \simeq \lambda=c / v$. However, the gravitational radius of the two-particle system (in the one-loop diagram) is $r_{g} \sim 2 G \cdot 2 h v / c^{4}$. Neglecting the non-spherical corrections, we can use the simplest case for estimations, the Schwarzschild metric, which gives for the redshifted frequency $\left(\bar{v}_{g} \equiv v_{g} / 2\right)$ :

$$
v=v_{0}\left(1-\frac{r_{g}}{r}\right)^{1 / 2} \simeq v_{0}\left(1-\frac{2 G}{c^{2}} \frac{2 h v}{c^{2}} \frac{v}{c}\right)^{1 / 2}=v_{0}\left(1-\frac{v^{2}}{\bar{v}_{g}^{2}}\right)^{1 / 2}
$$

Thus, the local frequency $v_{0}$ and the frequency $v$ in terms $t$ are related as:

$$
v_{0} \simeq \frac{v}{\left(1-v^{2} / \bar{v}_{g}^{2}\right)^{1 / 2}}, \quad v \simeq \frac{v_{0}}{\left(1+v_{0}^{2} / \bar{v}_{g}^{2}\right)^{1 / 2}} .
$$

It can be seen that even at $v_{0} \gg \bar{v}_{g}$ the observed frequencies of two particles in the oneloop diagram remain less than half of Planck frequency $v<\bar{v}_{g}$, i.e. total energy of the twoparticle system tends to Planck energy, which expresses the freezing of quantum fluctuations near $l_{g}$. For this reason, in the loop diagrams, integration over $v_{0}$ in the infinite interval $(0, \infty)$ should be replaced, using (4), by integration over $v$ in the finite interval $\left(0, v_{g}\right)$ with the upper limit $v_{g}$. Using (4), the differentials should be replaced as:

$$
d\left(v_{0}^{2}\right) \simeq \frac{d\left(v^{2}\right)}{\left(1-v^{2} / \bar{v}_{g}^{2}\right)^{2}}, \quad d\left(v^{2}\right) \simeq \frac{d\left(v_{0}^{2}\right)}{\left(1+v_{0}^{2} / \bar{v}_{g}^{2}\right)^{2}} .
$$

In the second term of (5), the integrals on $v_{0}$ explicitly converge due to the factor $v_{0}^{-4}$ and at $v_{0} \gg \bar{v}_{g}$ all $v$ in the integrals tend to constant $v \rightarrow \bar{v}_{g}$.

In fact, the gravitational regularization was earlier used implicitly as the Pauli-Villars regularization, when the propagators of particles were replaced by:

$$
\frac{1}{k^{2}-m^{2}} \rightarrow \frac{1}{k^{2}-m^{2}}-\frac{1}{k^{2}-M^{2}}=\frac{1}{k^{2}-m^{2}} \cdot \frac{1}{1-k^{2} / M^{2}} .
$$

In the one-loop diagrams, the replacement of both propagators as in (6) introduces the factor $k^{-4}$ in the integral, equivalent to the replacement in (5). 


\subsection{Simplified rules of gravitational regularization}

Thus, the gravitational freezing of all processes at the distance $l_{g}$ and the energy $\Lambda_{g}$ means that the events at the region $l \leq l_{g}$ or $\Lambda \geq \Lambda_{g}$ simply do not occur at a finite world time of distant observers $t<\infty$ and, therefore, they do not contribute to the amplitudes or the $S$ matrix. We can formulate the consequences for QFT of this restriction of general relativity as the first rule of the gravitational regularization:

1. In QFT, the gravitational freezing of processes at $l_{g}, \Lambda_{g}$ leads to the gravitational selfregularization of loop diagrams, and amplitudes include the contributions of only the distances $l>l_{g}$ and energies $\Lambda<\Lambda_{g}$.

The observed values of masses and charges are the sum of their bare values and loop corrections, and small inaccuracies in determining the loop corrections leads only to a small change in the bare values, which are already unknown. Therefore, to prove the finiteness and smallness of the loop corrections at such a regularization, it is not necessary to calculate them exactly, since it is enough to obtain upper limits for them.

The gravitational redshift of the frequencies of quanta in a loop diagram begins to decrease at the energies of quanta in terms of $t$ at distances of several $l_{g}$, for example, $3 l_{g}$. But a decrease in the energy of fluctuations effectively reduces the value of these corrections. Consequently, if we continue to use the standard dependence on the cutoff energy up to $\Lambda \sim \Lambda_{g}$ without gravity effects, then the absolute values of these corrections in the interval $3 l_{g} \geq r \geq l_{g}$ will be larger than their absolute values with effects of gravity. As the result, this gives the second rule:

2. Cutting off at $l_{g}$ or $\Lambda_{g}$ of the standard loop corrections without gravity gives upper limits ( $\Delta m_{\max }, \Delta e_{\max }^{2}$ ) for corrections with gravity effects.

In fact, the difference between the upper limit of the mass correction $\Delta m_{\max }$ and its true value $\Delta m$ (with gravity effects) is quite small: $\delta m=\Delta m_{\max }-\Delta m \ll \Delta m_{\max }$. This difference becomes added into the bare mass $m_{0}$, making it a lower limit for the bare mass $m_{0 \min }=m_{0}-\delta m$. The same is true for the bare charge $e_{0}^{2}: \delta e^{2}=\Delta e_{\max }^{2}-\Delta e^{2} \ll \Delta e_{\max }^{2}$ and $e_{0 \text { emin }}^{2}=e_{0}^{2}-\Delta e_{\max }^{2}$. Thus, we arrive at the third rule:

3. The physical mass $m$ and the physical charge $e^{2}$ are the sum of lower limits for bare values $m_{0 \min }, e_{0 \min }^{2}$ and upper limits for corrections $\Delta m_{\max }, \Delta e_{\max }^{2}$ :

$$
\begin{gathered}
m=m_{0}+\Delta m=\left(m_{0}-\delta m\right)+(\Delta m+\delta m)=m_{0 \text { min }}+\Delta m_{\max }, \\
e^{2}=e_{0}^{2}+\Delta e^{2}=\left(e_{0}-\delta e^{2}\right)+\left(\Delta e^{2}+\delta e^{2}\right)=e_{0 \text { min }}^{2}+\Delta e_{\max }^{2} .
\end{gathered}
$$

Further improvements will only lead to a slight redistribution between the bare values and the loop corrections. If the contribution of the upper limits to the total value of the loop correction is small and sufficient for the convergence of the perturbation theory series, then further refinements (with a more accurate inclusion of gravity) will only lead to a decrease in the absolute values of the corrections, which will lead to an even faster convergence of the perturbation theory series. 


\section{Gravitationally-regularized SM and quantum gravity}

\subsection{Upper limits of the loop corrections in QED}

In quantum electrodynamics (QED) the initial Lagrangian $L_{0}$ with bare fields and constants (by indices 0 ), depending on $\Lambda[1,2]$ :

$$
L_{0}(\Lambda)=\bar{\psi}_{0}\left(i \not \supset-m_{0}\right) \psi_{0}-\frac{1}{4}\left(F_{0}^{i k}\right)^{2}-e_{0} \bar{\psi}_{0} \gamma_{i} \psi_{0} A_{0}^{i}
$$

leads to the loop diagrams giving small corrections to the terms of $L_{0}$ :

$$
\begin{aligned}
& \delta L(\Lambda)=\bar{\psi}_{0}(i \not \supset-\Delta m) \psi_{0}\left(Z_{2}^{-1}-1\right)-\frac{1}{4}\left(F_{0}^{i k}\right)^{2}\left(Z_{3}^{-1}-1\right) \\
& -e_{0} \bar{\psi}_{0} \gamma_{i} \psi_{0} A_{0}^{i}\left(Z_{1}-1\right) .
\end{aligned}
$$

Here the constants $Z_{1}, Z_{2}, Z_{3}$ and $\delta m$ are also depend on $\Lambda$.

For the physical Lagrangian $L$, as the sum of (8) and (9):

$$
\begin{gathered}
L(\Lambda)=L_{0}(\Lambda)+\delta L(\Lambda)=Z_{2}^{-1} \bar{\psi}_{0}\left(i \not \supset-m_{0}-\Delta m\right) \psi_{0}- \\
-\frac{1}{4} Z_{3}^{-1}\left(F_{0}^{i k}\right)^{2}-Z_{1} Z_{2}^{-1} Z_{3}^{1 / 2} e_{0}\left(Z_{2} \bar{\psi}_{0} \gamma_{i} \psi_{0} \cdot Z_{3}^{-1 / 2} A_{0}^{i}\right),
\end{gathered}
$$

at the invariant cutoff energy $\Lambda=\Lambda_{g}$, we arrive at the gravitationally-regularized Lagrangian $L\left(\Lambda_{g}\right)$. By inserting into (10) the renormalized fields and constants:

$$
\psi=Z_{2}^{-1 / 2} \psi_{0}, A^{i}=Z_{3}^{-1 / 2} A_{0}^{i}, m=m_{0}+\Delta m, e=e_{0} Z_{1} Z_{2}^{-1} Z_{3}^{1 / 2}
$$

and equating $m$ and $e$, at the condition $\Lambda=\Lambda_{g}$, to their observed values, we arrive at the gravitationally-regularized finite physical Lagrangian $L$ :

$$
L=\bar{\psi}(i \not \supset-m) \psi-\frac{1}{4}\left(F^{i k}\right)^{2}-e \bar{\psi} \gamma_{i} \psi A^{i} .
$$

The constants $Z_{1}, Z_{2}, Z_{3}$ and $\delta m$, as show (10)-(12), are related with definite types of diagrams: $Z_{2}$ and $Z_{3}$ are the renormalization constants of the electron $\left(S_{c}\right)$ and photon $\left(G_{c}\right)$ propagators, respectively:

$$
\begin{array}{ll}
\left\langle\psi\left(x^{\prime}\right) \bar{\psi}(x)\right\rangle_{0}=Z_{2}^{-1}\left\langle\psi_{0}\left(x^{\prime}\right) \bar{\psi}_{0}(x)\right\rangle_{0}, & S_{c}=Z_{2}^{-1} S_{c}^{(0)}, \\
\left\langle A^{i}\left(x^{\prime}\right) A^{k}(x)\right\rangle_{0}=Z_{3}^{-1}\left\langle A_{0}^{i}\left(x^{\prime}\right) A_{0}^{k}(x)\right\rangle_{0}, & G_{c}=Z_{3}^{-1} G_{c}^{(0)} .
\end{array}
$$

The renormalized propagators at such finite regularization are expressed through the selfenergy diagrams. In the momentum representation for a photon, the latter is $\Pi\left(q^{2}\right)$, defined as $\Pi_{i k}\left(q^{2}\right)=\left(q_{i} q_{k}-q^{2} g_{i k}\right) \Pi\left(q^{2}\right)$, i.e. as the transverse part of the polarization operator. The correction to the electron mass is separated from the self-energy $\Sigma(p)$, where $S_{c}^{-1}=\left(S_{c}^{(0)}\right)^{-1}-\Sigma$,

$$
\Sigma(p)=\left[\Delta m-\left(Z_{2}^{-1}-1\right)+C(p)\right](p-m) .
$$


Then, the Ward identity $\partial \Sigma(p) / \partial p^{i}=-\Lambda_{i}(p, p)$, where $\Lambda_{i}\left(p, p^{\prime}\right)$ is the contribution of the vertex part, gives $Z_{1}=Z_{2}$. Inserting this into (11) allows to determine the physical charge: $e=e_{0} Z_{1} Z_{2}^{-1} Z_{3}^{1 / 2}=e_{0} Z_{3}^{1 / 2}$.

In QED with electrons and photons, the largest contribution to the renormalization constants give the one-loop diagrams with electrons in the intermediate state. The coupling constant $\alpha / 4 \pi \simeq 0.00058$ gives:

$$
\ln \frac{\Lambda_{g}^{2}}{m_{e}^{2}} \simeq 103.056, \quad A_{e}=\frac{\alpha}{4 \pi} \ln \frac{\Lambda_{g}^{2}}{m_{e}^{2}} \simeq 0.0598 .
$$

The polarization operator $\Pi\left(q^{2}\right)$ (at $q^{2} \rightarrow 0$ ) and $Z_{3}$, the renormalization constant of the photon propagator, have the values:

$$
\Pi(0) \simeq \frac{4}{3} A_{e} \simeq 0.0797 \simeq 7.97 \%, \quad Z_{3} \simeq 1-\Pi(0)=0.9203 .
$$

They give for the bare coupling constant $\alpha_{0}$ and a correction $\Delta\left(e^{2}\right)$ :

$$
\begin{gathered}
\alpha=Z_{3} \alpha_{0}, \quad \alpha_{0} \simeq \frac{\alpha}{0.92} \simeq \frac{1}{126.07} \simeq 0.0079, \\
\Delta\left(e^{2}\right)=\Pi(0) e^{2}=0.0797 e^{2} .
\end{gathered}
$$

The renormalization constants $Z_{1}$ and $Z_{2}$ are equal to:

$$
Z_{1}^{(1)}=Z_{2}^{(1)} \simeq\left(1+A_{e}\right)^{-1} \simeq 0.9436 .
$$

For electron, the bare mass $m_{e(0)}$ is about 0.85 of the physical mass $m_{e}$, and the difference between them is about $0.15 m_{e}$ :

$$
\begin{gathered}
m_{e(0)}=m_{e}\left(1+3 A_{e}^{(1)}\right)^{-1} \simeq 0.8479 m_{e}=0.4333 \mathrm{MeV}, \\
\Delta m_{e}=m_{e}-m_{e(0)} \simeq 0.1521 m_{e(0)}=0.0777 \mathrm{MeV} .
\end{gathered}
$$

Higher-order loop contributions in QED also remain small, and in all cases, gravitational regularization leads to rather small corrections to the observables. The factors $Z_{i}^{(n)}$ for $n$-loop diagrams can be calculated by different methods and the results show that in QED the perturbation theory is convergent.

\subsection{Upper limits of the loop corrections in SM}

In $\mathrm{SM}$, based on the symmetry group $S U(3) \times S U(2) \times U(1)$ and spontaneous symmetry breaking by scalar condensate [2], loop diagrams are finite at the regularization energy $\Lambda_{g}$. For gauge fields they are also small due to the logarithmic dependence on $\Lambda_{g}$ and the asymptotic freedom. In electroweak theory and QCD, the loop corrections practically reduce to modifications of the effective constants in the logarithmic factors (1). At ordinary distances, their coupling constants are greater than $\alpha$, but decrease at small distances and near $l_{g}$ become smaller $\alpha$. As a result, the series of perturbation theory also converge.

In SM, problems arise only in the scalar sector introduced for the spontaneous symmetry breaking. The loop integrals for the scalar field grow as $\Lambda^{n}, n>1$ which lead to finite but 
unacceptably large contributions to the observables of order $\Lambda_{g}^{n}$. This means that either the scalar particle is composite, and its field is effective, or there is a different mass generation mechanism modifying its present form in SM.

Thus, SM with gravitational regularization at $\Lambda_{g}$ becomes finite and consistent, since the predicted values of observables are finite and are in accordance with the observations, although with the last remark about the effective nature scalar field. The latter is the prediction of the gravitational regularization concerning the scalar field.

The triangle diagrams with fermion propagators and a pseudo-vector vertex form a special class of loop diagrams, which grow linearly with cutoff energy $\Lambda$ and for this reason lead to the chiral anomalies [2]. At the gravitational regularization, the contribution of such diagrams is finite, but of order $\sim \Lambda_{g}$, and therefore such large contributions are inadmissible.

For this reason, the criterion for the consistency of particle physics models has been and remains the mutual cancellation of chiral anomalies in the set of diagrams describing the observed processes. In SM, there is such cancellation of chiral anomalies, and this fact was one of the main selection criteria for both the quark model and the gauge field models [2]. Thus, the gravitational regularization at $\Lambda_{g}$ makes the situation with chiral anomalies in SM methodologically reasonable, since the theory operates with finite, although very large quantities which mutually cancel.

In the quark model, $\pi$-mesons are composite and are formed by a quark-antiquark pair, i.e. the pion field is effective and lose its meaning at small distances. Consequently, the chiral anomaly arising in this phenomenological model has nothing to do with ultraviolet divergences.

\subsection{Self-regularization of quantum gravity}

The regularization of the loop contributions by the proper external gravitational field, through the metric $g_{00}$ with cutoff at $\Lambda_{g}$, occurs also for the graviton field, quantized transverse components of the field, i.e. in quantum gravity. The gravitational time dilation in the system of particles only increases due to the presence of gravitons as a special kind of quanta.

The dimensional coupling constant in quantum gravity $G \sim 1 / \Lambda_{g}^{2}$ leads to power-law contributions from the loop diagrams with gravitons $\sim\left(\Lambda / \Lambda_{g}\right)^{2 L+2}$. However, unlike the scalar field, the effective dimensionless coupling constant $\left(\Lambda / \Lambda_{g}\right)^{2}<1$ remains small up to $\Lambda_{g}$. The one-loop contributions of order $\left(\Lambda / \Lambda_{g}\right)^{4}$ are rather small at $\Lambda<\Lambda_{g}$. Therefore, the contributions of multi-loop diagrams, where any additional loop adds two to the growth rate, are negligible and can be neglected.

Thus, for the loop contributions of quantum gravity the perturbation theory remains valid at $\Lambda<\Lambda_{g}$ and quantum gravity appears not only finite, but allows one to apply perturbation theory, which in addition converges. 


\section{Extended SM with gravitons and perspectives of generalization}

\subsection{The finiteness of theories of quantized fields and ESM}

Thus, the adaptation of QFT to the physical requirements of general relativity leads to its ultraviolet finiteness. But what confirms this key fact and what are its consequences?

The first evidence of the finiteness of the theories of known fields is agreement with experiment of SM. The predictions of QED are the most precise and well tested. QCD and electroweak theory are also mainly in agreement with experiments. The smallness of renormalized masses, charges, and magnetic moments of quarks and leptons also indicates the smallness of the loop corrections and the convergence of the perturbation theory, which indirectly confirms the gravitational regularization.

But, why does this confirm the gravitational regularization, and not some other? This is because gravitational regularization is required by the experimentally confirmed basic effect of general relativity, the gravitational time dilation, and therefore it is necessary condition that should have been respected initially. Moreover, it is the only regularization that naturally leads to a finite limit $\Lambda \rightarrow \Lambda_{g}$ for the regularization energy.

The consequences of the finiteness of QFT are various and non-trivial. Here we note only some of the main ones:

1) QFT becomes a finite and consistent physical theory;

2) an agreement between QFT and the general relativity is reached,

3) the long initial stage of the formation of QFT, when it contained internal contradictions in its foundations, is now finished;

4) there is no need to search and test very radical hypotheses and this makes the situation in particle physics sufficiently simpler;

5) at describing the structure of the vacuum of fields, the physical picture becomes simpler, since gravitational freezing excludes the hypotheses about a «hot» vacuum near Planck scale.

Quantum gravity becomes consistent and simpler than expected, since it reduces to the contributions at $\Lambda<\Lambda_{g}$, where it is enough to take the one-loop diagrams with gravitons, while higher order ones are insignificant.

After the gravitational regularization and considering the scalar sector as an effective field, SM and quantum gravity become ultraviolet-finite and the perturbation theory series converge. Therefore, SM and quantum gravity can be combined into one a general theory, Extended SM (ESM). At denoting by $G$ a local group of symmetry of graviton field, the total symmetry group of ESM fields takes the form $G \times S U(3) \times S U(2) \times U(1)$.

The specific form of the group $G$ should be clarified, since it can be separated from the group of general coordinate transformations by different ways, the results of which may differ. However, the standard diagram technique and the gravitational regularization remain applicable to the graviton field also.

\subsection{Simplification of unification models and perspectives for compositeness}

After the first successful unification in the electroweak theory, attempts to further unify the SM fields were natural and will remain one of the main areas of research in particle physics. Gravitational regularization further simplifies this situation by the prescription that now scalar fields, as effective, should not be one of the unified fields.

In addition, attempts to unify the three gauge fields into one were based on the hypothesis that at the energies of order $10^{15} \mathrm{GeV}$, the charges of three known gauge fields become equal. In fact, at the gravitational regularization at $\Lambda_{g}$, in the asymptotics of the three charges their curves intersect only in pairs and at three different points, the energy scales of which differ by 
several orders of magnitude. Thus, within the framework of SM, the Grand Unification of its interactions is either excluded or new facts are needed which are still unknown.

Another significant simplification of the situation in particle physics is the fact that the local QFT in 4-dimensional spacetime is sufficient to solve most of its former problems. Therefore, now to solve these problems there is no need to use hypotheses about unusual fundamental objects, as well as hypotheses about the existence of additional dimensions of space.

A special class of models are models based on the topologically nontrivial configurations of fields. Some of them, at a physically correct formulation of the solving problems, will develop further, since any accounting of nonlinear effects, especially such non-trivial ones, will improve the accuracy of calculations and can lead to the discovery of new phenomena.

The gravitational regularization opens up new perspectives explicitly for composite models. At first, the composite nature of the scalar boson is predicted, and moreover, at distances that will be available for experiments in the near future. In this regard, the theoretical problems of composite models become the most relevant and the main ones will probably be solved in the near future.

Models of composite particles will remain a perspective area for the further development of particle physics also from the point of view of the unification of interactions. The success of composite models would reduce the number of fundamental particles, which would simplify the unification models and restricts their versions. Although there are still no direct experimental facts about the substructure of quarks, leptons and gauge bosons, there are reasons stated above for the composite nature of the scalar particle.

Thus, a closer attention to composite models and a focus on their problems will lead to a situation that is natural from the point of view of historical tradition, when further progress in particle physics will be associated with a decrease in the number of primary particles, simplifying their models, and the unification of their interactions.

\section{Conclusion}

Previous attempts to include gravity on the Planck length $l_{g}$ consisted on the calculating of the contribution of the quantized component of the field, i.e. transverse gravitons leading to the gravitational analog of the Lamb shift only. Even at the inclusion of an external gravitational field, there was no clear understanding of the role and consequences of the gravitational time dilation, which dilates up to freezing all local processes.

According to general relativity, near $l_{g}$, the gravitational radius of quanta with Planck energy $\Lambda_{g}$, all processes freeze in asymptotically flat spacetime with world time $t$, where the $S$-matrix is constructed. The freezing of quantum fluctuations with extremal redshift of frequencies up to vanishing near $l_{g}$ leads to the gravitational self-regularization of quantum field.

The nonlinearity of non-Abelian gauge fields and quantum gravity makes gravitational effects even stronger, which means that freezing occurs faster and at larger distances, which even more strongly suppresses the contributions of high energies. The cutting of the integrals of the loop diagrams at the invariant energy $\Lambda_{g}$ gives upper limits for the loop corrections, which for the gauge fields of SM and quantum gravity turn out to decrease with increasing number of loops. This means the convergence of the perturbation theory series at $\Lambda<\Lambda_{g}$. This conclusion does not change with further refinement of the calculations, since the refinement means an earlier inclusion of the redshift effects than at $\Lambda_{g}$, which will only further reduce the loop contributions. 
Thus, QFT reconciled with general relativity, in particular with restrictions due to the gravitational time dilation at $l_{g}$, becomes ultraviolet-finite. Nonrenormalizable theories are also ultraviolet-finite and become consistent if their loop corrections remain small at cutting of their integrals at $\Lambda_{g}$. Most important of such cases is quantum gravity.

The hypothesis about the composite nature of SM particles becomes most relevant, since the scalar boson must be composite and the scalar field - effective. The SM with an effective scalar field together with quantum gravity, after gravitational regularization, form ESM, which is ultraviolet-finite and consistent.

\section{References}

1. Bjorken, J.D., Drell, S.D. Relat. Q. Mech; Relat. Quant. Fields, McG.-H. (1964-1965).

2. Itzykson, C. Zuber, J.-B. Quantum Field Theory, McG.-H. (1980).

3. Einstein A. J. Rad. u. Electr. 1907, 4, 411; Ann. d. Phys., 35, 898 (1911); 49, 769 (1916).

4. Rosenfeld, L. Z. Phys., 65, 589 (1930).

5. Bronstein, M. P. Phys. Z. d. Sowjet., 9, 140 (1936).

6. De Witt, B. Dynamical Theory of Groups and Fields. G.B. (1965).

7. 't Hooft, G. Nucl. Phys., B33, 173 (1971).

8. Markov, M. Sov. Phys. Uspekhi, 16, 587 (1974).

9. Fomin, P.I. Phys. Elem. Part. and At. Nucl., 7,3, 687 (1976);

Vilenkin, A.V., Fomin, P.I. Nuovo Cim., 45A, 1, 59 (1978).

10. Treder, H.-J. in „Relat., Quant. and Cosmol.“, p. 783, G.B.F (1979);

Borzeszkowski, von, H.-H., Treder, H.-J. Meaning of Q. Grav., D.R.P. (1988).

11. Faddeev, L. D. Sov. Phys. Usp., 25, 130 (1982).

12. Zakir, Z. Quant. and Grav. Phys., 1:002-7128 (2020). 